\title{
DISEÑO DE UN PROGRAMA DE EDUCACIÓN SOCIOEMOCIONAL PARA DOCENTES DE EDUCACIÓN BÁSICA
}

\author{
Maritza Palma Luengo \\ Universidad del Bío-Bío \\ mpalma@ubiobio.cl \\ Nelly Lagos San Martín \\ Universidad del Bío-Bío \\ nlagos@ubiobio.cl \\ Verónica López-López \\ Universidad de Concepción \\ veronicalopez@udec.cl
}

Recepción Artículo: 21 octubre 2021 Admisión Evaluación: 21 octubre 2021 Informe Evaluador 1: 22 octubre 2021 Informe Evaluador 2: 22 octubre 2021 Aprobación Publicación: 22 octubre 2021

\section{RESUMEN}

En el contexto educacional, se ha puesto especial énfasis en la integración de la dimensión emocional en los procesos de aprendizaje. Por lo mismo, este estudio busca diseñar e implementar a pequeña escala una metodología didáctica en el ámbito socioemocional para mejorar la eficacia de los procesos de enseñanza-aprendizaje en las escuelas. En el curso diseñado, participaron 80 profesores (60 mujeres; 20 hombres) de educación básica de cuatro establecimientos educativos; particular pagado (8), particular subvencionado (62) y municipales (4 rural y 6 urbano). El curso se dicta a través de la plataforma institucional de Adecca, utilizando zoom para las videoconferencias. Cada módulo se apoya y complementa con material escrito (libros, artículos y guías de aprendizaje) y material audiovisual (cápsulas, videos, páginas web). Se trata un curso de 45 horas pedagógicas, distribuidos en 5 módulos enfocados en lo teórico-practico y reflexivo, considerando además el acompañamiento a los docentes en el diseño e implementación de los programas educativos que ellos debieron desarrollar en sus establecimientos educativos, a través de la metodología proyectual. Es decir, los avances de los módulos se reflejan posteriormente en un proyecto educativo escolar que posteriormente se implementa en el contexto de aula. En cada módulo se revisó las tendencias de la dimensión emocional, brindando elementos conceptuales y prácticos para traer a la práctica de aula. El curso de formación fue valorado positivamente por los docentes participantes, especialmente por la oportunidad de contar con una instancia de trabajo colaborativo en la implementación de la educación socioemocional.

Palabras clave: formación continua; educación socioemocional; educación básica; formación docente. 


\title{
DISEÑO DE UN PROGRAMA DE EDUCACIÓN SOCIOEMOCIONAL PARA DOCENTES DE EDUCACIÓN BÁSICA
}

\begin{abstract}
Design of a socioemotional education program for elementary school teachers. In the educational context, special emphasis has been placed on the integration of the emotional dimension in learning processes. Therefore, this study seeks to design and implement on a small scale a didactic methodology in the socioemotional area to improve the effectiveness of the teaching-learning processes in schools. In the course designed, 80 teachers (60 women; 20 men) of basic education from four educational establishments participated; private paid (8), private subsidized (62) and municipal (4 rural and 6 urban). The course is delivered through Adecca's institutional platform, using zoom for videoconferences. Each module is supported and complemented with written material (books, articles and learning guides) and audiovisual material (capsules, videos, web pages). It is a course of 45 pedagogical hours, distributed in 5 modules focused on the theoretical-practical and reflective, considering also the accompaniment to the teachers in the design and implementation of the educational programs that they had to develop in their educational establishments, through the projective methodology. In other words, the progress of the modules is subsequently reflected in a school educational project that is then implemented in the classroom context. In each module, the trends of the emotional dimension were reviewed, providing conceptual and practical elements to bring to classroom practice. The training course was positively valued by the participating teachers, especially for the opportunity to have an instance of collaborative work in the implementation of social-emotional education.
\end{abstract}

Keywords: Continuing education; socio-emotional education; primary education; teacher training

\section{INTRODUCCIÓN}

En el contexto educacional, se ha puesto especial énfasis en reconocer la integración de la dimensión emocional en los procesos de aprendizaje, discusión que se ha manifestado tanto desde el trabajo docente (Cornejo et al., 2021) como en la experiencia estudiantil (Milicic et al., 2013; Agencia de Calidad de la Educación, 2018). Sobre este análisis, resulta tan necesario como urgente integrar y potenciar esta dimensión sinérgicamente en el aprendizaje, dentro de la formación continua del profesorado, dado que poco se ha discutido acerca de cómo implementar la educación socioemocional en el contexto de aula.

A partir de la convicción de que la educación debe preparar para la vida, en los últimos años se ha generado un movimiento a favor de promover, difundir y desarrollar la educación emocional como una innovación psicopedagógica. En este sentido, en el contexto de un proyecto de investigación regular interno de la Universidad del Bío-Bío, se diseña e implementa una innovación psicoeducativa para un curso de formación continua para profesores de educación básica del sistema escolar chileno. El curso se fundamenta en el desarrollo habilidades en el área de la educación socioemocional según el modelo pentagonal de Bisquerra y Pérez (2007), que a su vez se apoya con las orientaciones metodológicas de Cloud9world (2009-2021), que consta de un modelo sistémico y un enfoque derivado de la psicología positiva.

El modelo pentagonal de Bisquerra, considera competencias distribuidas en cinco grandes dimensiones que contribuyen a la educación emocional: la 1) conciencia emocional, cuyo eje central es la alfabetización emocional como punto de partida; la 2) regulación emocional entendida como la capacidad para canalizar las emociones; la 3) autonomía emocional en la cual se incluye el desarrollo de la autoestima como un elemento relevante; la 4) competencia social, incorporando en él, el desarrollo de habilidades sociales básicas y alternativas de resolución de conflictos y la 5) competencia para la vida y bienestar, bajo las cuales resulta importante el desarrollo de la felicidad, el fluir y el bienestar subjetivo.

El aporte del Programa de Cloud9world, se relaciona con los aspectos prácticos del curso, que favorece el aprendizaje socioemocional desde un modelo sistémico y un enfoque derivado de la psicología positiva, que permite una concepción amplia de educación emocional en la educación básica, basada en el desarrollo de la personalidad, en las competencias socio-emocionales, los aspectos motivacionales y las habilidades cognitivas.

Las características del proceso educativo impartido a los docentes, consistió en la combinación de clases teóricas, prácticas y reflexivas. Desde el punto de vista progresivo de los aprendizajes se enfatizó en la concep- 
tualización de cada competencia, así como en la importancia de su desarrollo, tanto a nivel personal como en el contexto de aula con los niños. Desde el punto de vista práctico, el programa cloud9world, permitió a los docentes conocer y apropiarse de las estrategias para organizarlas luego en un proyecto de trabajo en sus unidades educativas. El soporte virtual del curso también ofreció la oportunidad de contar con un repositorio de recursos y la posibilidad de generar actividades progresivas y secuenciales para la realización de los proyectos.

\section{OBJETIVOS DE LA INVESTIGACIÓN}

El estudio busca diseñar e implementar a pequeña escala una metodología didáctica en el ámbito socioemocional para mejorar la eficacia de los procesos de enseñanza-aprendizaje en las escuelas.

\section{MUESTRA Y/O PARTICIPANTES}

En el curso denominado Educación Emocional, participaron 80 profesores (60 mujeres; 20 hombres) de educación básica de cuatro establecimientos educativos; particular pagado (8), particular subvencionado (62) y municipales (4rural y 6 urbano).

\section{METODOLOGÍA Y/O INSTRUMENTOS UTILIZADOS}

El curso se dicta a través de la plataforma institucional de Adecca, utilizando zoom para las videoconferencias. Cada módulo se apoya y complementa con material escrito (libros, artículos y guías de aprendizaje) y material audiovisual (cápsulas, videos, páginas web). Adicionalmente, se cuenta con el acceso directo de los estudiantes a la plataforma de Cloud9word, a través de una cuenta exclusiva para el curso.

Se trata un curso de 45 horas pedagógicas, distribuidos en 5 módulos enfocados en lo teórico-practico y reflexivo, considerando además el acompañamiento a los docentes en el diseño e implementación de los programas educativos que ellos debieron desarrollar en sus establecimientos educativos, a través de la metodología proyectual (La Cueva, 1998). Es decir, los avances de los módulos se reflejan posteriormente en un proyecto educativo escolar que posteriormente se implementa en el contexto de aula (Figura 1). En cada módulo se revisó las tendencias de la dimensión emocional, brindando elementos conceptuales y prácticos para traer a la práctica de aula.

En cada módulo se plantearon 3 fases que consistió en una 1) Fase de contextualización del tema en cuestión cuyo objetivo es presentar los contenidos desde su modelo teórico; 3) Fase de Aplicación, orientado a que el profesorado desarrolle habilidades socioemocionales desde el ámbito personal y para el contexto de aula; y 3) Fase Reflexiva que busca que los docentes de Educación Primaria logren desarrollar competencias de trabajo reflexivo, critico, colaborativo y autónomo.

Figura 1.

Diseño del curso bajo la metodología de proyecto
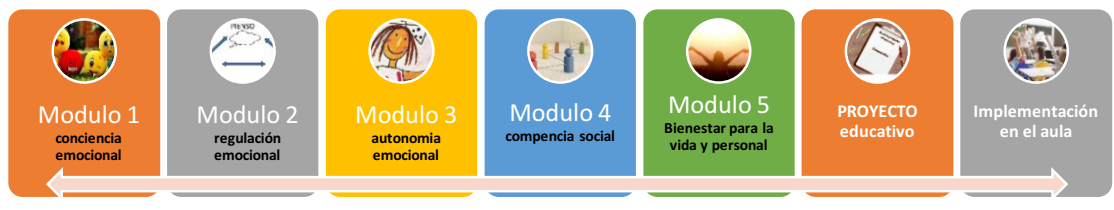

\section{RESULTADOS ALCANZADOS}

\section{Sobre el Proyecto Educativo}

El currículum educativo chileno desarrollado y administrado por el Ministerio de Educación, descansa sobre un modelo de asignaturas independientes, cada una con objetivos de aprendizaje propios al que se suma un conjunto de objetivos de aprendizaje transversales a considerar, que no se encuentran integrados con las asignaturas 


\section{DISEÑO DE UN PROGRAMA DE EDUCACIÓN SOCIOEMOCIONAL PARA DOCENTES DE EDUCACIÓN BÁSICA}

del currículum. Bajo esta metodología en cambio, se propone que los módulos sean interdependientes, complementando los objetivos de aprendizaje entre sí e integrando cada competencia como eje del proceso de aprendizaje. Esto fue abordado a través de la metodología de proyecto que se planteó transformar los objetivos de aprendizaje de contenidos aislados transformándolos en macro áreas temáticas bajo la figura de proyectos.

El aspecto más relevante que la metodología posee y que además es su eje conductor, se refiere al desarrollo de los aprendizajes basados en la motivación progresiva de los profesores que lograron mediante un paulatino involucramiento con la realidad, en la que aplican los contenidos del currículum de manera interdisciplinaria y de los módulos en sus propios contextos educativos. Estos avances conceptuales otorgados bajo la fase de contextualización fueron también apoyados con diversos recursos de cloud9word, los que fueron puestos a disposición de los profesores para que utilizaran en sus aulas con el fin de lograr apropiación de la metodología y al mismo tiempo generaran insumos para sus propuestas de proyectos.

Las actividades realizadas en los módulos fueron ampliamente aprovechadas por los equipos y a la vez altamente apreciadas por los profesores que por primera vez realizaban un curso bajo esta metodología. La mayoría coincide en haber vivido un proceso de aprendizaje de trabajo colaborativo y autónomo que, además, fue facilitado por el guía, quien se enfoca en dar retroalimentación o evaluación formativa constante para el logro de los proyectos.

Resultaron 4 proyectos de trabajo colaborativo, del total de 80 participantes, que en su mayoría corresponden a proyectos de colegios de tipo particular subvencionado y municipales. La mayoría de estos proyectos estuvieron focalizados en temáticas relacionadas a la conciencia y autonomía emocional, puesto que, para ellos, resulta más fácil ejercitar por primera vez, la alfabetización emocional y la autoestima como componente de la autonomía altamente conocida por los docentes.

\section{Sobre la implementación Del Proyecto Educativo}

La discusión final con los profesores en esta última fase resulta sincera y fructífera, y genera confianza en que el trabajo realizado a la fecha fue consistente y bien encaminado. Sin embargo, se hace ver el conflicto que tuvieron los profesores en la implementación del proyecto en los contextos educativos, debido a la exigencia horaria que la mayoría ha presentado bajo la modalidad virtual. Este inconveniente sumado a que aún no se cumplen los plazos de entrega que establece el curso, no permite visibilizar el impacto que éstos han tenido en los contextos educativos.

\section{DISCUSIÓN}

El curso de formación fue valorado positivamente por los docentes participantes, especialmente por la oportunidad de contar con una instancia de trabajo colaborativo en la implementación de la educación socioemocional, que no solo apoya en las necesidades emocionales del aula, como se vive en el contexto escolar, "la escuela es el lugar donde se aprende a vivir y a convivir, (...) el niño aprende a relacionarse con pares y con adultos en una interacción cotidiana e intensa" (Céspedes, 2018), sino que además fue estimulante para su propio bienestar, lo que coincide con lo señalado Costa, Palma y Salgado (2021), quienes señalan la importancia de la inteligencia emocional de los docente para la práctica pedagógica de aula.

\section{CONCLUSIONES}

Desde punto de vista de la innovación psicopedagógica, el diseño del curso resulta viable, dada la participación e interés progresivo en desarrollar las actividades propuestas en los módulos. Por lo mismo, se espera que esta investigación hecha con este enfoque teórico, practico y reflexivo, cree conocimiento teórico demostrando cómo el diseño y la estrategia metodológica proyectual utilizada, genere impacto en el aprendizaje en el contexto de estudio.

Sin embargo, en vista de la debilidad presentada en la última fase de implementación de los proyectos en contexto de aula, el equipo de investigación considera a futuro, el planteamiento de una nueva forma de evalua- 
ción del curso, involucrando el compromiso de los establecimientos con los aprendizajes logrados durante las capacitaciones de sus docentes, a fin de facilitar y hacer viable su aplicación.

En vista de lo anterior, sería relevante además contar con el impacto que los proyectos estarían generando en las comunidades educativas en las cuales se ha instalado este modelo, motivo por el cual el estudio se considera preliminar, pero viable y relevante en su diseño como curso socioemocional para la formación continua.

\section{AGRADECIMIENTOS}

Las autoras agradecen el financiamiento del proyecto de investigación DIUBB 2050238 IF/R de la Universidad del Bío-Bío.

\section{REFERENCIAS BIBLIOGRÁFICAS}

Agencia de Calidad de la Educación, (2018). Informe nacional de la calidad de la educación. http://archivos.agenciaeducacion.cl/INFORME_NACIONAL_DE_LA_CALIDAD_DE_LA_EDUCACION_2018.pdf

Céspedes, A. (2018). El estrés en niños y adolescentes: En busca del paraíso perdido. Santiago, Chile: Penguin Random House Grupo Editorial S. A. file:///C:/Users/sofia/Downloads/El-Estres-en-Ninos-y-AdolescenteCespedes.pdf

Cornejo Chávez, R., Vargas Pérez, S., Araya Moreno, R. y Parra Moreno, D. (2021). La educación emocional: paradojas, peligros y oportunidades. Revista Saberes Educativos, 6, 1-24. doi:10.5354/24525014.2021.60681

Bisquerra, R., y Pérez, N. (2007). Las competencias emocionales. Educación XXI, 10, 61-82.

Costa-Rodríguez, C., Palma-Leal, X. y Salgado-Farías, C. (2021). Docentes emocionalmente inteligentes. Importancia de la Inteligencia Emocional para la aplicación de la Educación Emocional en la práctica pedagógica de aula. Estudios pedagógicos, 47(1), 219-233. https://dx.doi.org/10.4067/S071807052021000100219

Cloud9world Chile (2009-2021). Programa de Habilidades Socioemocionales. https://cloud9worldchile.com/

La Cueva, A. (1998). La enseñanza por proyectos: ¿mito o reto? Revista Iberoamericana de Educación, (16), 165187. https://rieoei.org/historico/oeivirt/rie16a09.pdf

Milicic, N. Alcalay, L., Berger, C. y Álamos, P. (2013). Aprendizaje socioemocional en estudiantes de quinto y sexto grado: Presentación y evaluación de impacto del programa BASE. Ensaio: Avaliacao e Políticas Públicas en Educacao, 21(81), 645-666.

https://www.scielo.br/j/ensaio/a/hrH46QbCGY6zjz4XPhWVBtv/?format=pdf\&lang=es

Ministerio de Educación (2018). Bases Curriculares Primero a Sexto Básico. Santiago: Ministerio de Educación. Gobierno de Chile. http://www.curriculumenlineamineduc.cl/605/w3-article-21299.html 
